\title{
An explicit solution of Burgers' equation with special kinematic viscosity using decomposition technique
}

\author{
Bolujo Joseph Adegboyegun \\ School of Mathematics and applied Statistics, Faculty of Informatics, University of Wollongong, Australia
}

Email address:

bja998@uowmail.edu.au,bolurown2000@yahoo.com

\section{To cite this article:}

Bolujo Joseph Adegboyegun. An Explicit Solution of Burgers' Equation with Special Kinematic Viscosity Using Decomposition Technique. Pure and Applied Mathematics Journal. Vol. 2, No. 3, 2013, pp. 134-139. doi: 10.11648/j.pamj.20130203.14

\begin{abstract}
In this article, Adomian's Decomposition Method (ADM) is employed to approximate the solution of Burgers' equationwhich is one-dimensional non-linear differential equations in fluid dynamics. The exact solution for Burger's equation with low kinematic viscosity $\alpha<0.01$ does not exist in the literatures. Thus, we obtained an explicit solution for this special case. We compared our solution using ADM with the exact solution and the existing numerical solution while $\alpha \geq 0.01$. We found out that ADM converges very rapidly to the exact solution and performed better than the existing numerical method.
\end{abstract}

Keywords: Burger's Equation, Homotopy Perturbation Method, Adomian Decomposition Method

\section{Introduction}

Burger's equation is the scalar non-linear partial differential equation

$$
\begin{gathered}
\frac{\partial u}{\partial t}=\alpha \frac{\partial^{2} u}{\partial x^{2}}-u \frac{\partial u}{\partial x}, \quad u(x, 0)=\phi(x), \quad x \in X \subseteq \Re, t \geq 0, \\
X \times \mathfrak{R}^{+} \rightarrow \mathfrak{R}
\end{gathered}
$$

The parameter $\alpha \geq 0$ is referred to as the kinematic viscosity, due to the connection between the equation and the study of fluid dynamics. The equation was proposed as a model of turbulent fluid in motion. It is important in variety of applications, most notably as a simplification of the Novier-Stokes equation which model fluid dynamics. Theoretical and numerical solutions of Burger's like equations have attracted the interest of many Scientistsin the last few decades as a result of its wide applicability in Science and Engineering.Various methods for obtaining closed form solution to non-linear differential equations are described in the open literatures, such as the Painleve method, the Darboux transformation, the generalized separation of variables, the Tanh method, the Sine-Cosine method and the inverse scattering transform method [1]. Cole [2] studied the mathematical properties of Burgers' equation, the relationship between it and the turbulence and shock wave theories. He proposed an exact solution based on transformation, Benton et al[3] proposed different exact solutions of Burger's like equation and their classifications.It is noted that analytical methods for solving Burgers' like equations are very restricted and can only be suitable in very special cases; so they cannot be used to solve equations of numerous realistic scenarios.

However, many numerical algorithms for the solution of Burger's equation have been proposed by many authors. Varoglu et al [6] proposed an iso-parametric space B time finite-element method and utilized the hyperbolic differential equation associated with Burger's equation. Aksan et al [7] reduced Burger's equation to system of nonlinear ordinary differential equations by discretization in time and solved each non-linear ordinary differential equation by Galerkin method. Caldwell et al [8] used finiteelement approach by altering the size of the element at each stage using information from the previous steps. More recently,MiralBhaget et al (2013) proposed Homotopy perturbationmethod, this approacheliminated the drawbacks of the traditional perturbation method. M.HadiRafiee et al (2013) [9] proposed reconstruction of variational iteration method while B.B,Sanugi et al [4] used a Fourier series technique to transform the original Burgers' equation to system of ordinary differential equations with initial conditions which can be easily solved. We noted that all these numerical methods to approximate non-linear equations involve discretization, perturbation or transformation that obviously could change the physical structure of the equations and in most cases lead to poor 
convergence. The Adomian'sdecomposition method(ADM), first proposed by G.Adomian [10] for solving both linear and non-linear differential equations of all kinds has been widely used in the recent time. Unlike the traditional numerical methods, ADM solves non-linear problems without recourse to perturbation or discretization as practiced by the prior acts and the solution through ADM does not affected by error associated with aforementioned numerical tasks [10-14]. Computational experience has shown that ADM yields a very rapid convergence of the solution series and in most cases, only few iteration leads to very accurate solutions.

In this work, the reduced Burger's equation is solved by ADM approach. For kinematic viscosity smaller than 0.01 , the exact solution does not exist in the literatures because of low convergence of the infinite series. Our attention is focused on this special case and we provide an explicit solution that may serve as test for other numerical results. We also compared our results when $\alpha>0.01$ to the theoretical solutionsobtained by Cole [2] and the numerical solutions recently obtained by MiralBhagat et al [5] using Homotopyperturbation method. The equations are solved using only the initial conditions and the boundary conditions are used to justify the reliability of the method.

\section{Basic Ideas of Adomian's Decomposition Method}

We begin with anon-linearinhomogeneous differential equation in an operator form:

$$
L u+R u+N u=g(t)
$$

where $L=\frac{\partial}{\partial t}$ is the linear highest-ordered derivatives with respect to $t, R$ is the remainder term(s) of the linear operator and $N u$ represent the non-linear term(s). Thus we get

$$
L u=g(t)-R u-N u
$$

It was assumed that the inverse operator $L^{-1}$ exists and denoted by definite integral with respect to $t$. Operating the inverse operator on (1.2), we have;

$$
u=\phi(x)+L^{-1}(g(t))-L^{-1}(R u+N u)=u_{0}-L^{-1}(R u+N u)
$$

where $u_{0}$ is the term that arise from the initial condition $\phi(x)$ and the integration of the source term(s) $g(t)$. G.Adomian [10] assumed that the unknown solution $u(x, t)$ can be expressed as the sum of infinite series

$$
u(x, t)=\sum_{n=0}^{\infty} u_{n}(x, t)
$$

$$
\sum_{n=0}^{\infty} u_{n}(x, t)=u_{0}-L^{-1}\left(R \sum_{n=0}^{\infty} u_{n}+\sum_{n=0}^{\infty} A_{n}\right)
$$

where $A_{n}$ is the Adomian polynomial that represents the non-linear term $N u$ and $A_{n}\left(u_{0}, u_{1}, u_{2}, \ldots u_{n}\right)$ is defined by

$$
A_{n}=\frac{d^{n}}{n ! d \lambda^{n}}\left[N\left(\sum_{n=0}^{\infty} \lambda^{n} u_{n}\right)\right]_{\lambda=0}, \quad n=0,1,2
$$

\section{Analytical Solution of Burger's Equation}

Consider equation (1) with initial condition $u(x, 0)=\sin \pi x, \quad 0<x<1$

and homogeneous conditions $u(0, t)=u(1, t)=0$. By theHopf-Cole transformation [2]

$$
u=-2\left(\frac{\partial v}{v \partial x}\right)
$$

Equation (1) with initial and boundary conditions transforms to the linear heat equation;

$$
\begin{aligned}
& \frac{\partial v}{\partial x}=\alpha \frac{\partial^{2} v}{\partial x^{2}}, \quad v_{0}(x)=v(x, 0)=\exp \left[-(2 \alpha \pi)^{-1}(1-\cos \pi x)\right] \\
& v_{x}(0, t)=v_{x}(1, t)=0, \quad t>0
\end{aligned}
$$

where $v(x, t)$ is any solution of heat equation(8) and equation (7) is a solution of equation (1). Solving equation (6) using the method of separation of variables, we have;

$$
v(x, t)=a_{0}+\sum_{n=1}^{\infty}\left(a_{n} e^{-n^{2} \pi^{2} \alpha t} \cos (n \pi x)\right)
$$

where $a_{0}$ and $a_{n}(n=1,2,3 . .$.$) are Fourier$ coefficients and they can be evaluated as;

$a_{0}=\int_{0}^{1} \exp \left[-(2 \alpha \pi)^{-1}(1-\cos \pi x)\right] d x$

$a_{n}=2 \int_{0}^{1} \exp \left[-(2 \alpha \pi)^{-1}(1-\cos \pi x)\right] \cos (n \pi x) d x$

By using equation (7), we obtain the exact solution to equation (1) as;

$$
u(x, t)=\frac{2 \pi \alpha \sum_{n=1}^{\infty}\left(a_{n} e^{-n^{2} \pi^{2} \alpha t} n \sin (n \pi x)\right)}{a_{0}+\sum_{n=1}^{\infty}\left(a_{n} e^{-n^{2} \pi^{2} \alpha t} \cos (n \pi x)\right)}
$$




\section{ADM Algorithm for Burger's Equation}

Equation (1) can be written in an operator form;

$$
L_{l} u=\alpha L_{x x} u-u L_{x} u, \quad u(x, 0)=\sin (\pi x), x \in[0,1]
$$

where $L_{t}=\frac{\partial}{\partial t}, L_{x}=\frac{\partial}{\partial x}, \quad L_{x x}=\frac{\partial^{2}}{\partial x^{2}} \quad$ we choose $\phi(x)=\sin \pi x$ for the purpose of comparison with existing analytical and numerical results in $[10,11]$. Using equation(2-4), we have

$$
\sum_{n=0}^{\infty} u_{n}(x, t)=\sin \pi x-\sum_{n=0}^{\infty}\left[L^{-1}\left(u_{n} L_{x} u_{n}\right)-L^{-1}\left(\alpha L_{x x} u_{n}\right)\right]
$$

Where, $N(u)=u L_{x} u=\sum_{n=0}^{\infty} A_{n}$ and $R(u)=\alpha L_{x x} u$. We have from equation (12)

$$
\begin{aligned}
& \left.\begin{array}{c}
u_{0}=\sin \pi x \\
u_{n+1}=L^{-1}\left(\alpha L_{x x} u_{n}-A_{n}\right)
\end{array}\right\} \\
& u_{3}=\frac{-\pi^{3} t^{3} \sin (\pi x)}{3 !}\left[\alpha^{3} \pi^{3}+28 \alpha^{2} \pi^{2} \cos (\pi x)+5\right. \\
& u_{4}=\frac{\pi^{4} t^{4} \sin (\pi x)}{4 !}\left[\alpha^{4} \pi^{4}+120 \alpha^{3} \pi^{3} \cos (\pi x)\right. \\
& ++\frac{\pi^{4} t^{4} \sin (\pi x)}{4 !}[13-328 \alpha \pi \cos (\pi x)-114 \\
& \text { To obtain the other components, MATHEMATICA can } \\
& \text { be efficiently used.In practice, infinite terms of the series } \\
& \text { cannot be computed, we approximate the solution by } \\
& \text { computing the terms; } \Psi_{k}=\sum_{n=0}^{k-1} u_{n}(x, t) \text {. The accuracy of }
\end{aligned}
$$$$
u_{3}=\frac{-\pi^{3} t^{3} \sin (\pi x)}{3 !}\left[\alpha^{3} \pi^{3}+28 \alpha^{2} \pi^{2} \cos (\pi x)+51 \alpha \pi \cos (\pi x)-15 \alpha \pi+16 \cos (\pi x)^{3}-10 \cos (\pi x)\right]
$$$$
u_{4}=\frac{\pi^{4} t^{4} \sin (\pi x)}{4 !}\left[\alpha^{4} \pi^{4}+120 \alpha^{3} \pi^{3} \cos (\pi x)+606 \alpha^{2} \pi^{2} \cos (\pi x)^{2}-166 \alpha^{2} \pi^{2}+125 \cos (\pi x)^{4}\right]
$$$$
+\frac{\pi^{4} t^{4} \sin (\pi x)}{4 !}\left[13-328 \alpha \pi \cos (\pi x)-114 \cos (\pi x)^{2}+568 \alpha \pi \cos (\pi x)^{3}\right]
$$
the method can be further improved if more-terms approximations of the solution are computed. As far as
The first few terms of Adomian polynomials $A_{n}$ can be determined using equation (5) with $u_{n x}=L_{x} u_{n}=\frac{\partial u_{n}}{\partial x}$

$A_{0}=u_{0} u_{0 x}$

$A_{1}=u_{1} u_{0 x}+u_{0} u_{1 x}$

$A_{2}=u_{2} u_{0 x}+u_{1} u_{1 x}+u_{0} u_{2 x}$

$A_{3}=u_{3} u_{0 x}+u_{2} u_{1 x}+u_{1} u_{2 x}+u_{0} u_{3 x}$

$A_{4}=u_{4} u_{0 x}+u_{3} u_{1 x}+u_{2} u_{2 x}+u_{0} u_{4 x}+u_{1} u_{3 x}$

$A_{5}=u_{5} u_{0 x}+u_{4} u_{1 x}+u_{3} u_{2 x}+u_{1} u_{4 x}+u_{2} u_{3 x}+u_{0} u_{5 x}$

The solution of equation (11) by using equations (13)(14), can be written as follows;

$$
\begin{gathered}
u_{0}=\sin \pi x \\
u_{1}=-\pi t \sin (\pi x)[\alpha \pi+\cos (\pi x)] \\
u_{2}=\frac{\pi^{2} t^{2} \sin (\pi x)}{2}\left[\alpha^{2} \pi^{2}+6 \alpha \pi \cos (\pi x)+3 \cos (\pi x)^{2}-1\right]
\end{gathered}
$$

accurate results are concerned, computational experience has shown that they can be easily obtained by computing the first ten-terms of the series.

The unknown solution $u(x, t)$ can therefore be obtained as $u(x, t)=u_{0}+u_{1}+u_{2}+u_{3}+u_{4}+$. . .

$$
\begin{aligned}
u(x, t) & =\sin \pi x-\pi t \sin (\pi x)[\alpha \pi+\cos (\pi x)]+\frac{\pi^{2} t^{2} \sin (\pi x)}{2}\left[\alpha^{2} \pi^{2}+6 \alpha \pi \cos (\pi x)+3 \cos (\pi x)^{2}-1\right] \\
& -\frac{\pi^{3} t^{3} \sin (\pi x)}{3 !}\left[\alpha^{3} \pi^{3}+28 \alpha^{2} \pi^{2} \cos (\pi x)+51 \alpha \pi \cos (\pi x)-15 \alpha \pi+16 \cos (\pi x)^{3}-10 \cos (\pi x)\right] \\
& +\frac{\pi^{4} t^{4} \sin (\pi x)}{4 !}\left[\alpha^{4} \pi^{4}+120 \alpha^{3} \pi^{3} \cos (\pi x)+606 \alpha^{2} \pi^{2} \cos (\pi x)^{2}-166 \alpha^{2} \pi^{2}+125 \cos (\pi\right. \\
& +\frac{\pi^{4} t^{4} \sin (\pi x)}{4 !}\left[13-328 \alpha \pi \cos (\pi x)-114 \cos (\pi x)^{2}+568 \alpha \pi \cos (\pi x)^{3}\right]+\ldots .
\end{aligned}
$$




\section{Comparison of ADM Solutions with Exact and Numerical Solutions}

In order to show the applicability and reliability of the ADM techniques, we have applied the approach to the problem given by equation (11) whose exact solution exists and is given by Cole [2] in terms of infinite series. To show the accuracy of the method for moderate size of kinematic viscosity values, we give the comparison withanalytical solutions from infinite series of Cole [2] and numerical solutions from Homotopy perturbation method of MiralBhagat et al [5] for $\alpha=1$ at different values of $t$ in tables $1,2 \& 3$. The tables show that ADM solutions are in good agreement with the exact solutions and it converges rapidly with half a dozen of terms. It is obvious from the tables that solutions using ADM performs better than the results of MiralBhagat et al using Homotopy perturbation method. Thus, ADM can help to overcome the problems caused by the shortage of analytical methods for the computation of solutions to nonlinear differential equation

Table 1. Analytical solutions of Burger's equation at different value of $t$ and $\alpha=1$ Cole [2]

\begin{tabular}{cccc}
\hline $\mathbf{x}$ & $\mathbf{t}=\mathbf{0 . 0 1}$ & $\mathbf{t}=\mathbf{0 . 0 0 1}$ & $\mathbf{t}=\mathbf{0 . 0 0 0 1}$ \\
\hline 0 & 0.0000 & 0.0000 & 0.0000 \\
0.1 & 0.2724 & 0.3037 & 0.3072 \\
0.2 & 0.5210 & 0.5797 & 0.5861 \\
0.3 & 0.7228 & 0.8012 & 0.8098 \\
0.4 & 0.8575 & 0.9455 & 0.9549 \\
0.5 & 0.9096 & 0.9962 & 1.0054 \\
0.6 & 0.8714 & 0.9473 & 0.9551 \\
0.7 & 0.7451 & 0.8039 & 0.8098 \\
0.8 & 0.5430 & 0.5822 & 0.5860 \\
0.9 & 0.2859 & 0.3052 & 0.3070 \\
1.0 & 0.0000 & 0.0000 & 0.0000 \\
\hline
\end{tabular}

Table 2. Numerical solutions using Adomian's method at different value of $t$ and $\alpha=1$

\begin{tabular}{cccc}
\hline $\mathbf{x}$ & $\mathbf{t}=\mathbf{0 . 0 1}$ & $\mathbf{t}=\mathbf{0 . 0 0 1}$ & $\mathbf{t}=\mathbf{0 . 0 0 0 1}$ \\
\hline 0 & 0 & 0 & 0 \\
0.1 & 0.2728 & 0.3039 & 0.3073 \\
0.2 & 0.5212 & 0.5798 & 0.5864 \\
0.3 & 0.7230 & 0.8016 & 0.8099 \\
0.4 & 0.8578 & 0.9456 & 0.9552 \\
0.5 & 0.9098 & 0.9964 & 0.9999 \\
0.6 & 0.8718 & 0.9480 & 0.9548 \\
0.7 & 0.7453 & 0.8040 & 0.8089 \\
0.8 & 0.5434 & 0.5834 & 0.5867 \\
0.9 & 0.2856 & 0.3055 & 0.3079 \\
1.0 & 0.0000 & 0.0000 & 0.0000 \\
\hline
\end{tabular}

Table 3. Numerical solutions using Homotopy method at different value of $t$ and $\alpha=1$ MiralBhagat [5]

\begin{tabular}{cccc}
\hline $\mathbf{x}$ & $\mathbf{t}=\mathbf{0 . 0 1}$ & $\mathbf{t}=\mathbf{0 . 0 0 1}$ & $\mathbf{t}=\mathbf{0 . 0 0 0 1}$ \\
\hline 0 & 0 & 0 & 0 \\
0.1 & 0.2732 & 0.3051 & 0.3086 \\
0.2 & 0.5214 & 0.5806 & 0.5871 \\
0.3 & 0.7218 & 0.7996 & 0.8081 \\
0.4 & 0.8546 & 0.9408 & 0.9500 \\
0.5 & 0.9058 & 0.9902 & 0.9990 \\
0.6 & 0.8684 & 0.9426 & 0.9502 \\
0.7 & 0.7441 & 0.8035 & 0.8084 \\
\hline
\end{tabular}

\begin{tabular}{llll}
\hline 0.8 & 0.5438 & 0.5835 & 0.5878 \\
0.9 & 0.2870 & 0.3069 & 0.3088 \\
1.0 & 0.0000 & 0.0000 & 0.0000 \\
\hline
\end{tabular}

In the case $\alpha<0.01$, it is not practical to compute the analytical solution at these values due to slow rate of convergence of the infinite series and thus the analytical solution is unknown. However, this special case was mentioned by MiralBhagat et al [5] but an attempt was not made to compute address this special case. Thus, it is extremely valuable that a numerical solution for this special case be added to the literature. However, we don't claim finality on this result since none existed before for comparison. But, we strongly believe that our result could be a standard test for comparison and can be used to validate other numerical solutions for more complicated cases where no exact solutions exist.

Table 4. Adomian's method solutions obtained at $\alpha=0.001$ with different value of $t$

\begin{tabular}{cccc}
\hline $\mathbf{X}$ & $\mathbf{t}=\mathbf{0 . 0 1}$ & $\mathbf{t}=\mathbf{0 . 0 0 1}$ & $\mathbf{t}=\mathbf{0 . 0 0 0 1}$ \\
\hline 0 & 0 & 0 & 0 \\
0.1 & 0.2996 & 0.3081 & 0.3089 \\
0.2 & 0.5696 & 0.5859 & 0.5876 \\
0.3 & 0.7836 & 0.8065 & 0.8089 \\
0.4 & 0.9209 & 0.9481 & 0.9508 \\
0.5 & 0.9681 & 0.9969 & 0.9997 \\
0.6 & 0.9208 & 0.9481 & 0.9507 \\
0.7 & 0.7836 & 0.8065 & 0.8088 \\
0.8 & 0.5696 & 0.5859 & 0.5876 \\
0.9 & 0.2996 & 0.3081 & 0.309 \\
1 & 0 & 0 & 0 \\
\hline
\end{tabular}

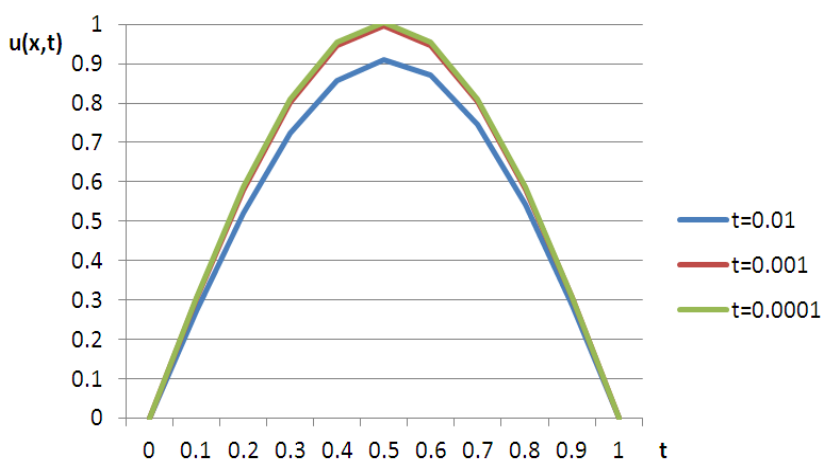

Figure 1. Analytical solution of Burger's equation at different value of $t$ with $\alpha=1$

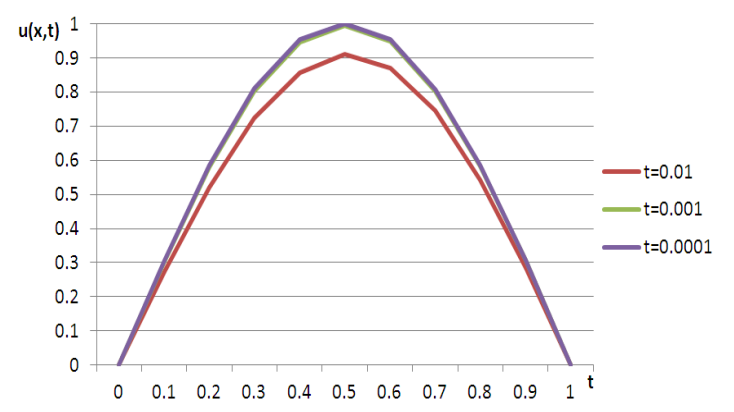

Figure 2. ADM solution of Burger's equation at different value of $t$ with $\alpha=1$ 


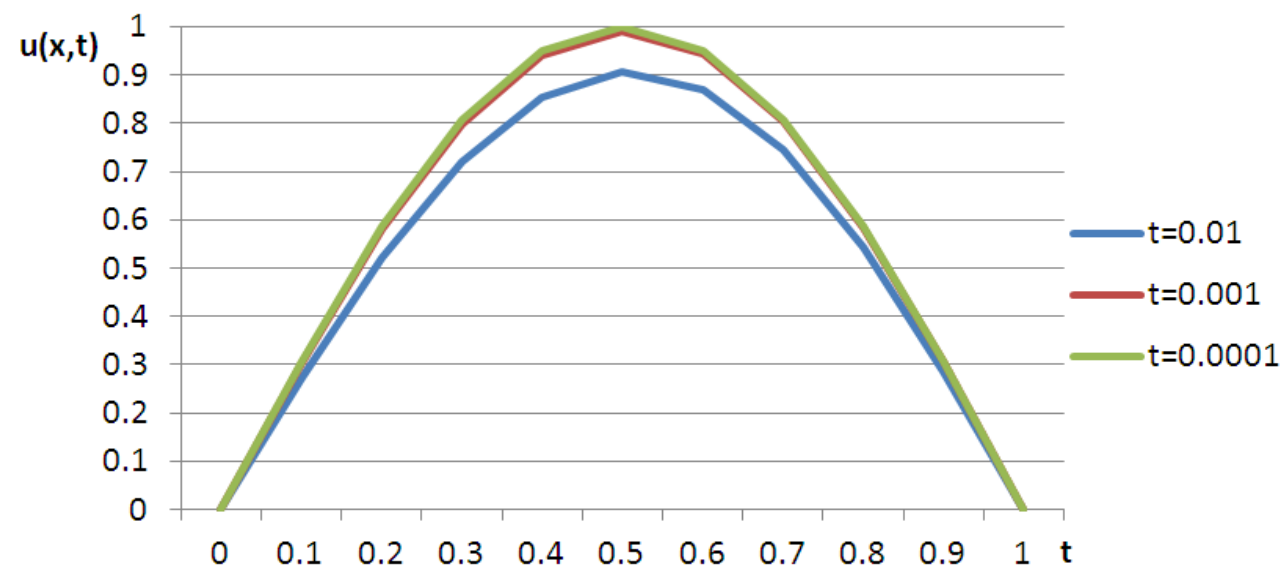

Figure 3. Homotopy perturbationsolution [5] of Burger's equation at different value of t with $\alpha=1$

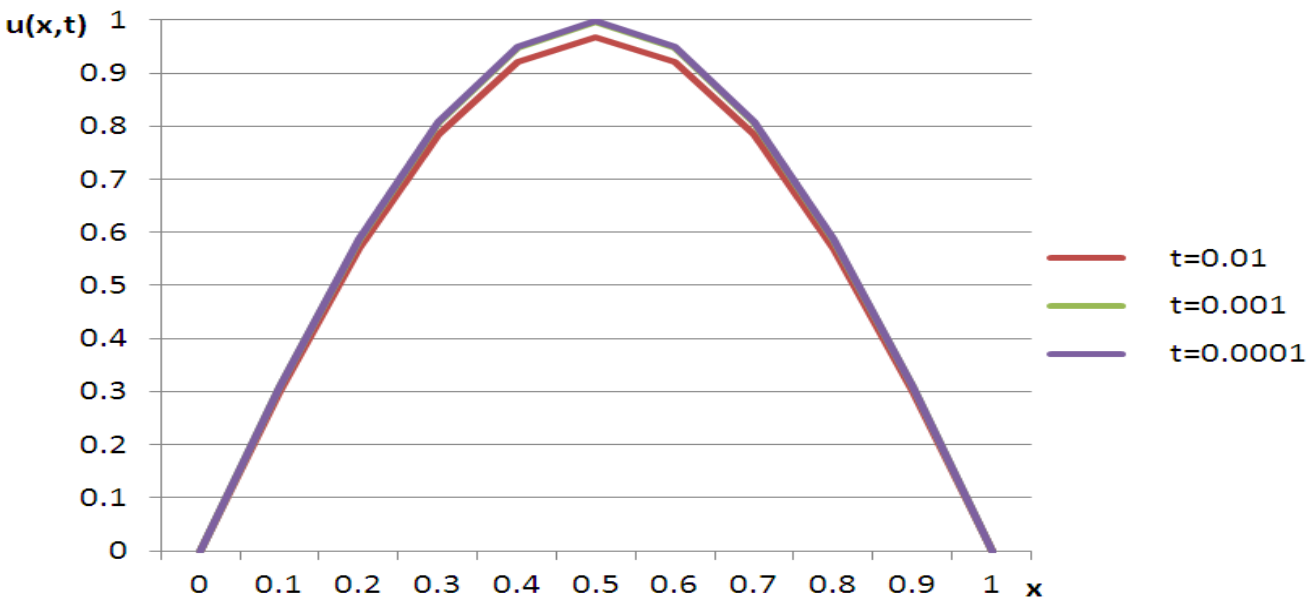

Figure 4. ADM solution of Burger's equation at different value of t with $\alpha=0.001$

\section{Conclusions}

The Adomian Decomposition Method has been successfully applied to approximate solution of Burgers' equation. For moderately small kinematic viscosity $\alpha \geq 0.01$, the Adomian's approach provides high accuracy while using a small number of terms and the results are better when compared with Homotopy perturbation method.We have also added to the literatures a numerical result for $\alpha=0.001$ where neither analytical nor numerical result existed before. We remark that ADM is a powerful mathematical method which solves all kinds of mathematical problem without recourse to perturbation, discretization, transformation or linearization as practiced by other conventional numerical methods.

\section{References}

[1] L. Debnath, Nonlinear Partial Differential Equations for Scientists and Engineers, Second ed., Birkauser, Broston, 2004.
[2] Cole J.D, On a quasilinear parabolic equation occurring in aerodynamics, Quart. Appl. Math., 9 pp 225-236

[3] Benton et al, A table of solution of the one dimensional Burgers' equation, Quart, Appl. Math., 30, pp 195-212

[4] B.B Sangi et al, A Fourier method for the solution of the nonlinear advection problem, Appl. Math., (14) (1988), 385389

[5] MiralBhaget et al (2013), Explicit solution of Burgers' and generalized Burgers' equation usingHomotopy perturbation method. IJAET.Vol.,6, issue 1, pp 179-188

[6] Varoglu et al, Space B time finite elements incorporating characteristics for Burgers' equation,Int. J. Num. Math. Eng., 16 pp 171-184

[7] Alksan, E.N., A.A. Zdes, and T.A-Zis, (1972), A numerical solution of Burgers' equation based on least squares approximation. AppliedMath. Comput., 176, pp 270-279

[8] Caldwell J., Wanless P, and Cook A.E (1981), A finite element approach to Burger's equation, Applied MathModel, 5, pp 189-193

[9] M. HadiRafiee et al (2013), Approximate solution to Burgers' equation using reconstruction of Variational iteration method. Appl., Math. 2013, 3 (2): 45-49 
[10] G. Adomian, Solving frontier problems of physics. The Decomposition Method. Kluer,Boston; 1994

[11] Wazwaz A.M, A new algorithm for calculating Adomian polynomials for nonlinearoperators, Appl. Math Comp 2000; 11: $35-51$

[12] G. Adomian, A review of the decomposition method in applied mathematics. J. Math Anal Appl. 1988; 135:501-544
[13] Adomian, G. and Rach, R. (1983b), "Nonlinear stochastic operators", Journal of Mathematical Analysis and Applications, vol. 91, no. 2, pp. 94-10

[14] B.J Adegboyegun and E.A Ibijola. (2012), A Comparison of Adomian's DecompositionMethod and Picard Iteration Method In Solving Non-linear Differential Equations. Global Journals Inc. (US), Vol. 12 Issue 7; 1 (2012): 37-42. 